\title{
Role of sweet and other flavours in liking and disliking of electronic cigarettes
}

\author{
Hyoshin Kim, ${ }^{1}$ Juyun Lim, ${ }^{2}$ Stephanie S Buehler, ${ }^{3}$ Marielle C Brinkman, ${ }^{3}$ \\ Nathan M Johnson, ${ }^{3}$ Laura Wilson, ${ }^{3}$ Kandice S Cross, ${ }^{3}$ Pamela I Clark ${ }^{4}$
}

${ }^{1}$ Battelle Public Health Center for Tobacco Research, Battelle Memorial Institute, Seattle, Washington, USA

${ }^{2}$ Department of Food Science and Technology, Oregon State University, Corvallis, Oregon, USA

${ }^{3}$ Battelle Public Health Center for Tobacco Research, Battelle Memorial Institute, Columbus, $\mathrm{OH}$, USA

${ }^{4} S$ chool of Public Health, University of Maryland, College Park, Maryland, USA

\section{Correspondence to}

Dr Hyoshin Kim, Battelle Public Health Center for Tobacco Research, Battelle Memorial Institute, 1100 Dexter Avenue N., Suite 400, Seattle, WA 98109, USA; kimh@battelle. org

Received 1 June 2016 Accepted 15 September 2016 Published Online First 5 October 2016

\section{ABSTRACT}

Objective To examine the extent to which the perception of sweet and other flavours is associated with liking and disliking of flavoured electronic cigarettes (e-cigarettes).

Methods 31 participants (13 females/18 males; 12 sole/19 dual users) vaped 6 commercially available flavours of blu Tanks: Classic Tobacco (CT), Magnificent Menthol (MM), Cherry Crush (CC), Vivid Vanilla (VV), Piña Colada (PC) and Peach Schnapps (PS); all 'medium' strength, $12 \mathrm{mg} / \mathrm{mL}$ nicotine concentration. For each flavoured e-cigarette, participants first rated liking/ disliking on the Labeled Hedonic Scale, followed by perceived intensities of sweetness, coolness, bitterness, harshness and specific flavour on the generalised version of the Labeled Magnitude Scale. The psychophysical testing was conducted individually in an environmental chamber.

Results PC was perceived as sweetest and liked the most; CT was perceived as least sweet and liked the least. Across all flavours, liking was correlated with sweetness $(r=0.31)$, coolness $(r=0.25)$, bitterness $(r=-0.25)$ and harshness $(r=-0.29$, all $p<0.001)$. Specifically, liking was positively correlated with sweetness of PS $(r=0.56, p=0.001)$ and $P C(r=0.36$, $\mathrm{p}=0.048)$; and with coolness of MM, CT and VV $(r=0.41-0.52, p<0.05)$. In contrast, harshness was negatively correlated with liking for CC, PC and PS $(r=0.37-0.40, p<0.05)$. In a multivariate model, sweetness had the greatest positive impact on liking followed by coolness; harshness had the greatest negative impact on liking.

Conclusions Our findings indicate that bitterness and harshness, most likely from nicotine, have negative impacts on the liking of e-cigarettes, but the addition of flavourants that elicit sweetness or coolness generally improves liking. The results suggest that flavours play an important role in e-cigarette preference and most likely use.

\section{INTRODUCTION}

The present research addresses the issue of how flavour perceptions, specifically of sweet flavour, influence liking and disliking of electronic cigarettes (e-cigarettes). Earlier research on conventional tobacco cigarettes revealed that cigarette sweetness is closely related to ratings of satisfaction and pleasantness. ${ }^{1}$ Currently, the addition of 'characterising' flavours to cigarettes, with the exception of menthol, is banned due to their appeal to youth. ${ }^{2-6}$ Therefore, no artificial or natural flavour (other than tobacco or menthol) or an herb or spice that is a characterising flavour of the tobacco product or tobacco smoke can be contained in a cigarette. ${ }^{7}$
However, this ban would not automatically extend to e-cigarettes under the new deeming regulations that became effective on 8 August $2016 .{ }^{8}$ Under the deeming regulation, the Food and Drug Administration (FDA) has given an extended, different compliance period for flavoured tobacco products, compared with non-flavoured products, in order to examine potential impacts of flavours on tobacco appeal and use. Consideration was given to non-combusted flavoured products, such as e-cigarettes, to weigh the risks and benefits to the general population and subpopulations, and specifically the increased/decreased likelihood that (1) non-users will initiate use (eg, youth population) and (2) current and former smokers will stop cigarette smoking. The present research focuses on e-cigarette flavours perception and its influence on the liking and disliking of e-cigarettes.

Flavours are a prominent aspect of e-cigarette merchandising. A 2014 study reported that there are more than 7000 unique flavours of e-cigarettes, ${ }^{9}$ including not just the traditional flavours of 'tobacco' and 'menthol', but ones having names associated with sweet items, such as desserts and candies. Previous research shows that sweet flavours can play an important role in e-cigarette use behaviours. $^{10-15}$ In a recent survey of adult ecigarette users, many of whom adopted e-cigarettes to stop or reduce cigarette smoking, a large proportion later adopted sweet flavours after an e-cigarette initiation using tobacco-like flavours; more than $61 \%$ of the respondents reported that they preferred sweet e-cigarette flavours. ${ }^{10}$ E-cigarette use is also growing in the youth population, which has shown a preference for sweet flavours as well. Over $70 \%$ of middle and high school students who smoke e-cigarettes have tried sweet-flavoured eliquids, and more than half prefer to vape with sweet flavours. ${ }^{14}$ Thus, there is a legitimate concern that flavoured e-cigarettes could lead to experimentation with and adoption of e-cigarettes by young non-smokers. Tempering this concern is a growing appreciation that the same characteristics which make e-cigarettes attractive to non-smokers also appeal to current cigarette smokers, in particular those motivated to quit smoking. The increased adoption of sweet-flavoured e-cigarette products has the potential to result in an increase in youth e-cigarette initiation, and a reduction in the use of combustible tobacco products.

To gain a better understanding of the relationship between sweet flavours and liking/disliking of e-cigarettes, we adopted psychophysical methods that are commonly used in the studies of foods and consumer products. This is in contrast to previous 
studies, ${ }^{10} 1415$ which used survey techniques to examine the relationship between perceived sweetness of e-cigarette and its usage. Recent work by Rosbrook and Green ${ }^{16}$ illustrates the effectiveness of the psychophysical approach in demonstrating sensory effects of menthol and nicotine in e-cigarettes. In the present research, we applied a similar approach to investigate the role of flavours in hedonic responses to e-cigarettes in an adult population.

\section{METHODS}

Participants

Thirty-one experienced sole and dual e-cigarette users were recruited from the Columbus, Ohio area. Participants were recruited by advertisements on Craigslist and in local college and city newspapers, flyers in local vape shops, and word-of-mouth. Participants had to (1) be healthy; (2) $>18$ years of age; (3) have been vaping for $\geq 1$ month; (4) use an e-cigarette liquid with at least a medium nicotine strength $(9-12 \mathrm{mg} / \mathrm{mL})$ and (5) be willing to use blu eCigs of various flavours during a laboratory session. Dual users also had to satisfy the following inclusion criteria: (1) currently smoking cigarettes; (2) have smoked cigarettes within the past 30 days and (3) have been regularly smoking cigarettes for $\geq 1$ year. Dual users had to use e-cigs at least 3 days a week to be eligible, while sole e-cigarette users had to vape every day. Sole e-cigarette users could also not currently be using other tobacco products, such as little cigars, waterpipes, smokeless tobacco, etc. Exclusion criteria across both user groups included: (1) mouth or throat problems that would keep the participant from vaping comfortably; (2) health problems that would keep the participant from tasting or smelling normally; (3) respiratory allergies; (4) a history of pulmonary disease or asthma; (5) claustrophobia; (6) being in the process of trying to quit vaping; and (7) being pregnant, breast feeding or trying to become pregnant. Participants were asked to abstain from eating, vaping and smoking (for dual users) 2 hours prior to their scheduled visit. Participant eligibility was confirmed at the beginning of the visit, and the informed consent was read and signed before the session began. This study was approved by the Battelle Institutional Review Board. Participants were compensated at the end of the session (\$75).

\section{Materials and equipment}

The test e-cigarettes, blu Tanks, were provided to each participant for vaping in the laboratory session. The blu Tanks are a cigalike e-cigarette with a closed, non-refillable system and do not contain the wicking material typical of cigalike products. Given the myriad of e-cigarette styles, flavours and brands available, the blu Tanks brand was chosen as an exemplar of a selfcontained, standardised product configuration and e-liquid formula. The following six flavours were tested: two conventional tobacco flavours (Classic Tobacco (CT) and Magnificent Menthol (MM)), and four non-tobacco flavours (Cherry Crush (CC), Piña Colada (PC), Peach Schnapps (PS) and Vivid Vanilla (VV)). These represent all of the available regular flavours sold for blu Tanks and they cover a range of flavour profiles currently available in commercial e-liquids. Each flavour was provided to the participant in the medium nicotine strength $(12 \mathrm{mg} / \mathrm{mL})$. According to the manufacturer, these flavours contained a 60:40 ratio of vegetable glycerin (VG):propylene glycol. Although we did not experimentally confirm this ratio, in a now-discontinued version of these blu eCig flavours (purchased in January 2014), we previously found no VG in the e-liquid. ${ }^{17}$ All blu Tanks flavour cartridges used in this study were purchased online through the blu eCig website in August 2015, and the tanks were coupled with the blu PLUS + battery system.

\section{Testing chamber}

All vaping sessions took place in a sealed, self-contained chamber with a controlled ventilation system of six air changes per hour $(2500 \mathrm{~L} / \mathrm{min})$. This ventilation sufficiently cleared the room of any secondhand vapour, and prevented unnecessary exposure of laboratory staff when entering the chamber. The chamber has a door and observation windows on three sides and was designed specifically for tobacco smoking sessions with human participants.

\section{Procedures}

Each session consisted of two parts: training on hedonic and intensity scales, and data collection. Both parts were conducted on a one-on-one basis in the sealed chamber described previously.

Training on scales: Prior to vaping the test e-cigarettes, participants were trained on how to use both hedonic and intensity scales. The Labeled Hedonic Scale (LHS) and the general version of the Labeled Magnitude Scale (gLMS) were used to measure liking/disliking and perceived intensity of flavour attributes. The LHS ${ }^{18-20}$ is a bipolar category-ratio scale bounded by 'most disliked sensation imaginable' at the bottom and 'most liked sensation imaginable' at the top, with its intermediate hedonic labels (ie, like or dislike: slightly, moderately, very much, extremely) spaced according to their empirically determined semantic magnitudes ${ }^{18}$ along with 'neutral' at its midpoint. The scale was displayed on a paper ballot, and participants were instructed to rate the degree of liking or disliking of each e-cigarette flavour by placing a slash mark at the appropriate place on the scale. In order to provide a broad context of hedonic sensations and allow the participants to understand how to use the LHS, the participants were asked to rate their liking or disliking of a list of 15 remembered or imagined sensations (eg, the taste of your favourite chocolate, the smell of bad body odour).

The gLMS ${ }^{21-23}$ is also a category-ratio scale bounded by 'no sensation' at the bottom and 'strongest imaginable sensation of any kind' at the top, with intermediate intensity labels (ie, weak, moderate, strong and very strong) spaced quasi-logarithmically according to their empirically determined semantic magnitudes. The distances between descriptors were determined by experimental outcomes that measured the strengths of the descriptors. $^{21-23}$ The scale was displayed on a paper ballot and participants were instructed to rate various flavour attributes they experienced for each e-cigarette flavour by placing a slash mark at the appropriate place on the scale. As with the LHS, in order to provide a broad context of sensations and best prepare the participant for using this device, the participants were asked to rate 15 remembered or imagined sensations (eg, the sweetness of milk, the heat of sipping boiling hot tea, the weight of a feather in your hand). The data from the training session were used for our internal validity check to identify substantial outliers among participants (eg, participant with all responses being no sensation or strongest imaginable) and all participants' responses fell within a normal range.

Data collection: Once a participant was sufficiently trained on the use of the scales, the participant was asked to go through a palate cleansing procedure. He or she first rinsed the mouth with deionised water $\left(37 \pm 0.5^{\circ} \mathrm{C}\right)$ and spit out the water. This was repeated two more times. The participant was then instructed to eat an unsaltine cracker (ie, a saltine cracker with 
no salt) followed by rinsing the mouth vigorously three more times with deionised water, spitting out the water after each rinse. The participant was then instructed to take four puffs from the first e-cigarette flavour. After taking the puffs, the participant first rated liking/disliking of the sample on the LHS and then rated the perceived intensities of five attributes (ie, sweetness, bitterness, harshness, coolness, own flavour) on five separate scales. For the 'own flavour', the participant was asked to describe the flavour of the e-cigarette in his/her own words and then rate its intensity. After completing the assessment of the first e-cigarette flavour, the participant repeated the palate cleansing procedure and then exited the chamber to take a 3-5 min break. The latter procedure was employed to clear his or her nasal passage. After the allotted break time, the participant re-entered the chamber and was given the second e-cigarette flavour. The entire process was repeated for the second e-cigarette flavour, and again four more times until all six e-cigarette flavours had been vaped and assessed. The flavours were assigned in random order to each participant using a Williams design.

\section{Data preparation and analysis}

Ratings for the LHS were measured from the bottom of the scale in millimetres and were translated into a range from -100 to +100 . Ratings for the gLMS were also measured from the bottom of the scale in millimetres. While hedonic responses on the LHS tend to be normally distributed across participants, ${ }^{18} 19$ intensity ratings on the gLMS tend to be log-normally distributed. $^{21} 22$ Therefore, the gLMS intensity ratings, but not the hedonic ratings, were log-transformed prior to statistical analyses.

To examine differences between e-cigarette flavours in hedonic ratings and sensory attribute ratings, one-way analysis of variance (ANOVA) with Tukey's honest significant difference tests were performed. To examine linear relationships between liking/disliking and sensory attributes, Pearson product-moment correlations were calculated. To examine the relative effects of flavour attributes on hedonic ratings, regression analyses were performed. As the same participants provided ratings multiple times for the same flavour in our study, their rating data were correlated. So as not to inflate the significance level, our regression models were estimated by correcting for intraclass correlation caused by non-independent observations. All statistical analyses were performed using Stata V.14.1.

\section{RESULTS}

\section{Participants' characteristics}

Participants' characteristics are reported in table 1 . Of the 31 participants, $58 \%$ were male and $84 \%$ were white. Average age was 34 years. About one-third of participants were dual users, averaging 18.1 years of cigarette smoking; five were mentholcigarette users. Dual users had used e-cigarettes for 26 months on average whereas sole users for 20 months; the average nicotine strength was $20.6 \mathrm{mg} / \mathrm{mL}$ for dual users and $17.2 \mathrm{mg} / \mathrm{mL}$ for sole users. All of the participants reported using a variety of e-cigs, including cigalikes, vape pens, and box and tank mods. All of the participants had tried flavoured e-liquids other than tobacco flavour, with the majority $(80 \%)$ preferring flavoured e-liquids when vaping. Some participants preferred to vape traditional tobacco flavours $(n=6)$ or menthol $(n=3)$. Reported flavours were: fruit $(n=13)$; vanilla $(n=6)$ and other such as bubble gum and absinthe $(n=3)$. Based on the Penn State Electronic Cigarette Dependence Index, ${ }^{24}$ about 20\% $(n=6)$
Table 1 Characteristics of participants $(\mathrm{N}=31)$

\begin{tabular}{|c|c|}
\hline Characteristic & $\mathrm{N}(\%)$ \\
\hline \multicolumn{2}{|l|}{ Gender } \\
\hline Male & $18(58 \%)$ \\
\hline Female & $13(42 \%)$ \\
\hline Age (in years) & $33.6 \pm 10.9$ \\
\hline \multicolumn{2}{|l|}{ Race } \\
\hline White & $26(84 \%)$ \\
\hline Black & $5(16 \%)$ \\
\hline Education (in years) & $14.2 \pm 1.9$ \\
\hline \multicolumn{2}{|l|}{ E-cigarette use status } \\
\hline Dual user & $12(38.7 \%)$ \\
\hline Sole user & $19(61.3 \%)$ \\
\hline \multicolumn{2}{|l|}{ Dual user } \\
\hline Non-menthol cigarette user & $7(58.3 \%)$ \\
\hline Menthol cigarette user & $5(41.6 \%)$ \\
\hline Length of combustible cigarette smoking (in years)* & $18.1 \pm 12.6$ \\
\hline \multicolumn{2}{|l|}{ Length of e-cigarette use (in months) } \\
\hline Dual user* & $26 \pm 14$ \\
\hline Sole user* & $20 \pm 11$ \\
\hline \multicolumn{2}{|l|}{ Nicotine strength (mg/mL) } \\
\hline Dual user* & $20.6 \pm 5.7$ \\
\hline Sole usert & $17.2 \pm 6.5$ \\
\hline \multicolumn{2}{|l|}{ Favourite e-cigarette flavour category $\ddagger$} \\
\hline Fruit (eg, berries, peach, cherry, watermelon) & $13(42.0 \%)$ \\
\hline Tobacco & $6(19.4 \%)$ \\
\hline Vanilla & $6(19.4 \%)$ \\
\hline Menthol & $3(9.6 \%)$ \\
\hline Other (black magic, bubble gum, capp and absinthe) & $3(9.6 \%)$ \\
\hline \multicolumn{2}{|l|}{ Penn State Electronic Cigarette Dependence Index } \\
\hline High dependence & $6(19.4 \%)$ \\
\hline Medium dependence & $14(45.2 \%)$ \\
\hline Low dependence & $5(16.1 \%)$ \\
\hline Not dependent & $6(19.4 \%)$ \\
\hline
\end{tabular}

were classified as not e-cigarette dependent and half $(n=14)$ as medium dependent.

\section{Liking/disliking and sensory attributes of e-cigarette flavours}

Figure 1A displays the mean hedonic (liking/disliking) ratings of the six e-cigarette flavours. PC was liked significantly more than CT $(p<0.05)$ with other flavours (MM, CT, PS and VV) being placed in between the two flavours in terms of the degree of liking. The scatter plot reveals the distributions of individuals' hedonic ratings for the six flavours. With the exception of CC, hedonic ratings of all five flavours were more or less symmetric with centre values as the means. An asymmetric distribution of hedonic ratings for $\mathrm{CC}$ indicates high disliking by a few participants.

Figure 1B shows the mean log-intensity ratings of sweetness, coolness, bitterness, harshness and own flavour in response to each of the six e-cigarette flavours. One-way ANOVAs confirmed a significant main effect of e-cigarette flavours on sweetness $(\mathrm{F}=14.56, \mathrm{p}<0.0001)$, coolness $(\mathrm{F}=11.96, \mathrm{p}<0.00001)$ and bitterness $(\mathrm{F}=3.56, \mathrm{p}<0.01)$, but not on harshness $(\mathrm{F}=1.77, \mathrm{p}>0.05)$ and own flavour $(\mathrm{F}=2.11, \mathrm{p}>0.05)$. As expected, the four non-tobacco flavoured e-cigarette samples 
Figure 1 (A) Mean hedonic ratings \pm SEMs (top left) and scatter plot of hedonic ratings (top right) of the six e-cigarette flavours with $12 \mathrm{mg} / \mathrm{mL}$ nicotine concentration across all participants $(\mathrm{N}=31)$. $\mathrm{CT}, \mathrm{MM}, \mathrm{CC}, \mathrm{PC}$, PS and VV represent for Classic Tobacco, Magnificent Menthol, Cherry Crush, Piña Colada, Peach Schnapps and Vivid Vanilla. Letters on the right $y$-axis represent semantic labels of the LHS. (B) $\log _{10}$ means \pm SEMs of intensity ratings of sweetness, coolness, bitterness, harshness and own flavour across all participants. Letters on the right $y$-axis represent semantic labels of the gLMS. Different letters $(a-c)$ indicate significant differences between e-cigarette flavours (Tukey's honest significant difference test, $\mathrm{p}<0.05)$. BD, barely detectable; $\mathrm{DE}$, dislike extremely; DVM, dislike very much; DM, dislike moderately; DS, dislike slightly; e-cigarette, electronic cigarette; gLMS, general version of the Labeled Magnitude Scale; LHS, Labeled Hedonic Scale; LS, like slightly; LM, like moderately; LVM, like very much; LE, like extremely; $M$, moderate; $S$, strong; VS, very strong; W, weak.
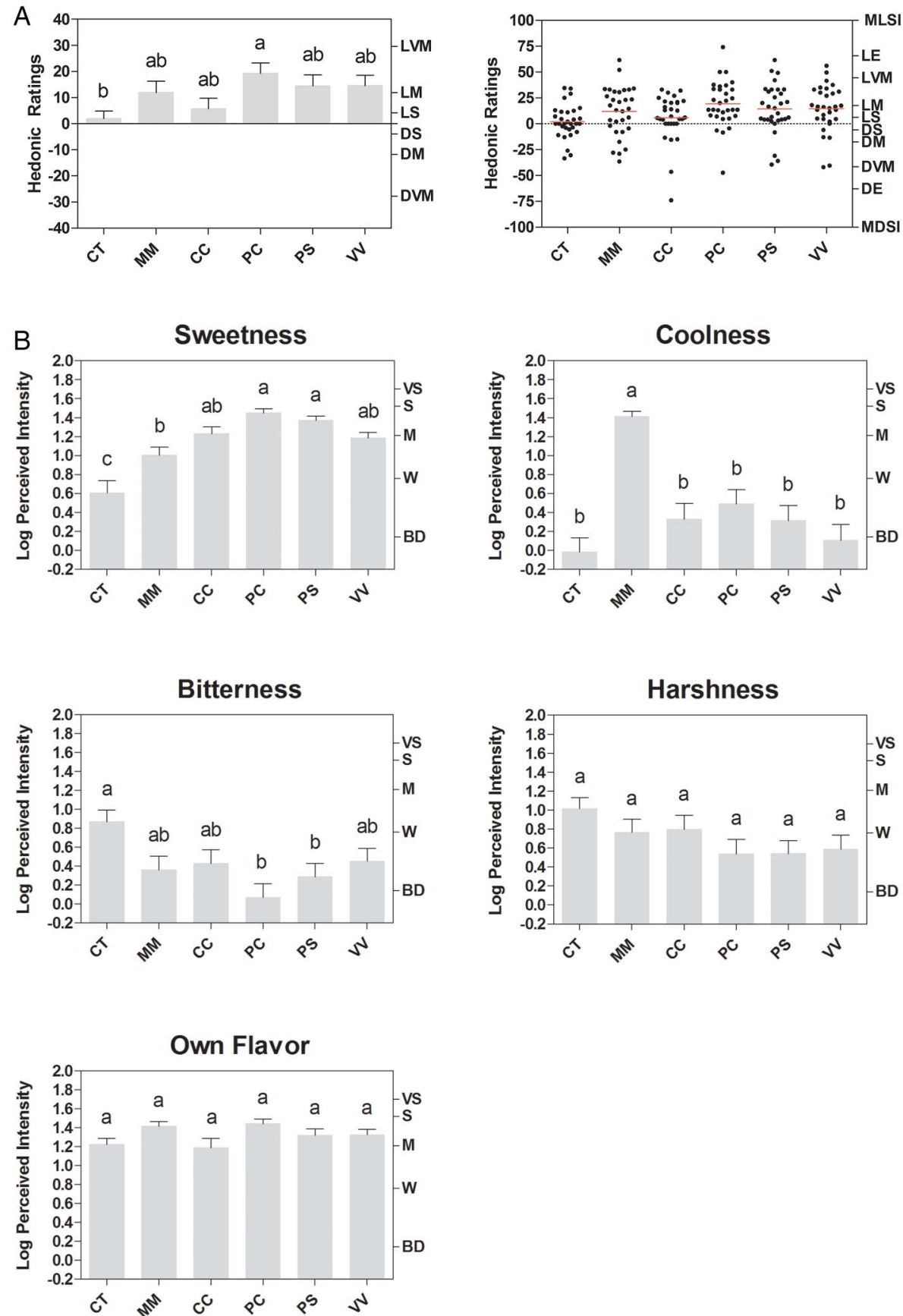

(CC, PC, PS and VV) were rated significantly sweeter than CT, and $\mathrm{MM}$ was rated significantly higher in cooling sensation than all other flavours. For bitterness, CT was rated significantly higher than PC and PS $(p<0.05)$. Trends in the data indicated that CT was also the harshest, although harshness ratings across the e-cigarette flavours were not significant. Hedonic and sensory ratings in figure $1 \mathrm{~A}, \mathrm{~B}$ collectively indicate that $\mathrm{PC}$ was perceived as sweetest and liked the most; CT was perceived as least sweet and liked the least.

\section{Role of sensory attributes in e-cigarette flavour liking}

Table 2 shows the univariate relationship between hedonic ratings and the perceived intensities of each sensory attributes for the six e-cigarette flavours. Hedonic ratings were significantly positively correlated for sweetness for PC $(r=0.36$, $\mathrm{p}<0.05)$ and PS $(\mathrm{r}=0.56, \mathrm{p}<0.05)$. Similar positive but weaker correlations were found for CT $(r=0.32, p=0.08), \mathrm{MM}$ $(\mathrm{r}=0.34, \mathrm{p}=0.06)$ and $\mathrm{VV}(\mathrm{r}=0.28, \mathrm{p}=0.13)$. For CC, no meaningful relationship was found for hedonic ratings with sweetness. Hedonic ratings were also positively correlated with coolness for all six flavours, but this relationship was only found to be statistically significant for CT, MM and VV ( $\mathrm{r}=0.41-0.52$, $\mathrm{p}<0.05$ ). In contrast, harshness ratings were significantly negatively correlated with hedonic ratings for CC, PC and PS $(\mathrm{r}=0.37-0.40, \mathrm{p}<0.05)$. Bitterness was also negatively correlated with hedonic ratings across all flavours, but not significantly so. 'Own flavour' was positively related to hedonic ratings, most notably for $\mathrm{MM}(\mathrm{r}=0.60, \mathrm{p}<0.0001)$, where participants commonly described it as 'refreshing' and 'minty'. A statistically significant positive correlation between hedonic ratings and 'own flavour' intensity ratings was also observed for 
Table 2 Correlations of hedonic ratings and specific attribute for six e-cigarette flavours $(\mathrm{N}=31)$

\begin{tabular}{lllllll}
\hline $\begin{array}{l}\text { Sensory } \\
\text { attribute }\end{array}$ & $\mathrm{CT}$ & $\mathrm{MM}$ & $\mathrm{CC}$ & $\mathrm{PC}$ & $\mathrm{PS}$ & $\mathrm{VV}$ \\
\hline Sweetness & 0.32 & 0.34 & -0.13 & $\mathbf{0 . 3 6}$ & $\mathbf{0 . 5 6}$ & 0.28 \\
& $\mathrm{p}=0.075$ & $\mathrm{p}=0.058$ & $\mathrm{p}=0.503$ & $\mathrm{p}=0.048$ & $\mathrm{p}=0.001$ & $\mathrm{p}=0.132$ \\
Coolness & $\mathbf{0 . 4 4}$ & $\mathbf{0 . 5 2}$ & 0.09 & 0.29 & 0.11 & $\mathbf{0 . 4 1}$ \\
& $\mathrm{p}=\mathbf{0 . 0 1 3}$ & $\mathrm{p}=0.002$ & $\mathrm{p}=0.632$ & $\mathrm{p}=0.117$ & $\mathrm{p}=0.544$ & $\mathrm{p}=0.021$ \\
Bitterness & -0.12 & -0.29 & -0.25 & -0.34 & -0.12 & -0.05 \\
& $\mathrm{p}=0.511$ & $\mathrm{p}=0.107$ & $\mathrm{p}=0.171$ & $\mathrm{p}=0.063$ & $\mathrm{p}=0.512$ & $\mathrm{p}=0.804$ \\
Harshness & -0.001 & -0.03 & -0.40 & -0.39 & -0.37 & -0.19 \\
& $\mathrm{p}=0.995$ & $\mathrm{p}=0.871$ & $\mathrm{p}=0.026$ & $\mathrm{p}=0.032$ & $\mathrm{p}=0.040$ & $\mathrm{p}=0.301$ \\
Own flavour & 0.13 & $\mathbf{0 . 6 0}$ & -0.11 & 0.31 & 0.30 & $\mathbf{0} .38$ \\
& $\mathrm{p}=0.489$ & $\mathrm{p}=0.000$ & $\mathrm{p}=0.549$ & $\mathrm{p}=0.086$ & $\mathrm{p}=0.106$ & $\mathrm{p}=0.034$ \\
\hline
\end{tabular}

Correlation coefficients and $p$ values are shown; bold characters indicate statistically significant values at $\mathrm{p}<0.05$.

CC, Cherry Crush; CT, Classic Tobacco; E-cigarette, Electronic Cigarette; MM,

Magnificent Menthol; PC, Piña Colada; PS, Peach Schnapps; VV, Vivid Vanilla.

$\mathrm{VV}(\mathrm{r}=0.38, \mathrm{p}=0.03)$. The most often listed descriptors were 'caramel' and 'coffee drinks' for VV.

To examine the general effects of sensory attributes on the potential liking and disliking of e-cigarettes, we regressed sensory attributes on hedonic ratings after aggregating the data of the six flavours in one data set. When a single attribute was considered as a factor (see table 3, models 1-4), sweetness and coolness showed their positive contributions, while bitterness and harshness showed negative contributions to liking and disliking of the six e-cigarettes flavours. When sweetness was considered as a sole factor, $9.6 \%$ of the total variation in hedonic ratings was explained, while coolness explained $6.4 \%$ of the variation in hedonic ratings to six flavours (see table 3, model 1 vs 2). Next, we added three attributes to the regression models to estimate their relative impacts on hedonic ratings (see table 3, models 5-6). By adding more sensory attributes, the total variation explained in hedonic ratings was improved to $20.8 \%$. When all four attributes were included in one regression model (model 7), however, the total variation explained in hedonic ratings was not improved any further $\left(\mathrm{R}^{2}=21.7 \%\right)$. Note that the coefficients of bitterness and harshness were no longer statistically significant in the model because harshness and bitterness ratings were strongly correlated $(r=0.62, p<0.001)$. Regardless of the model employed, the regression coefficient of sweetness exceeded that of coolness (see table 3, models 5-7). All of the regression models were estimated by correcting for intraclass correlation caused by non-independent observations.
Roles of other factors in e-cigarette flavour liking

We explored the roles of age, gender and race on the relationships between hedonic rating and sensation intensity ratings. None were significant factors in the regression models. We also included variables of participants' dual-use status, menthol smoker status, as well as e-cigarette dependence grouping status in the model, but none of the variables were statistically significant. Inclusion of those variables did not change the relationships between hedonic rating and intensity ratings described earlier.

\section{DISCUSSION}

Our findings suggest that flavours play an important role in liking and disliking of e-cigarettes. First and foremost, the results show that bitterness and harshness are negatively associated with liking of e-cigarettes, while sweetness and coolness are positively associated with liking. In other words, our findings suggest that perceived bitterness and harshness reduce the liking of e-cigarette flavours, but the addition of flavourants that elicit sweetness and coolness improves the liking. In addition, our findings indicate that the impact of sweetness on liking is greater than the impact of coolness. These findings imply that the role of sweet flavours in e-cigarettes is potentially significant in attracting current sole e-cigarette users and dual e-cigarette and cigarette users. As indicated by positive correlations between sweetness and liking/disliking ratings (see table 2), sweetness ratings of e-cigarette flavours are associated with higher liking, even in tobacco (CT) and menthol (MM) flavours. These results are not surprising, given that liking for sweet substances is innate, and that sweetness can suppress bitterness. ${ }^{25} 26$

Our findings also provide evidence that e-cigarette flavours with cooling attributes appeal to adult e-cigarette users. It is well established that menthol produces a cooling sensation along with a minty flavour. ${ }^{27}$ Previous studies have demonstrated that menthol has the effect of masking bitter taste and reducing harshness/irritation of cigarette smoke and nicotine. ${ }^{16}{ }^{28-33}$ Furthermore, menthol tends to increase liking. ${ }^{16}$ The improved sensory appeal of menthol cigarettes is greatest for youth, women and African-Americans, ${ }^{30}{ }^{33-35}$ and the positive associations of menthol flavour in e-cigarettes demonstrated in this study suggest that these subpopulations may be at risk for greater rates of e-cigarette initiation and use.

In contrast to conventional cigarettes, characterising flavours are still permitted in other tobacco products under the new deeming rule, including e-cigarettes. ${ }^{7}$ While the exact mechanism of how sweet flavours increase or reduce liking of tobacco products is unclear, the present study provides the first

Table 3 Regression coefficients with p values examining the effects of sensory attribute intensity ratings on hedonic ratings in different models

\begin{tabular}{|c|c|c|c|c|c|c|c|}
\hline Sensory attribute & Model 1 & Model 2 & Model 3 & Model 4 & Model 5 & Model 6 & Model 7 \\
\hline Sweetness & $\begin{array}{l}13.37 \\
p=0.000\end{array}$ & - & - & - & $\begin{array}{l}10.50 \\
p=0.005\end{array}$ & $\begin{array}{l}10.41 \\
p=0.007\end{array}$ & $\begin{array}{l}10.32 \\
p=0.007\end{array}$ \\
\hline Coolness & - & $\begin{array}{l}6.06 \\
p=0.007\end{array}$ & - & - & $\begin{array}{l}4.49 \\
p=0.038\end{array}$ & $\begin{array}{l}4.73 \\
p=0.032\end{array}$ & $\begin{array}{l}4.74 \\
p=0.027\end{array}$ \\
\hline Bitterness & - & - & $\begin{array}{l}-7.09 \\
p=0.011\end{array}$ & - & $\begin{array}{l}-7.07 \\
p=0.003\end{array}$ & - & $\begin{array}{l}-3.27 \\
p=0.244\end{array}$ \\
\hline Harshness & - & - & - & $\begin{array}{l}-8.05 \\
p=0.024\end{array}$ & - & $\begin{array}{l}-8.25 \\
p=0.013\end{array}$ & $\begin{array}{l}-6.18 \\
p=0.121\end{array}$ \\
\hline $\mathrm{R}^{2}$ & $9.6 \%$ & $6.4 \%$ & $6.5 \%$ & $8.1 \%$ & $18.8 \%$ & $20.8 \%$ & $21.7 \%$ \\
\hline
\end{tabular}


psychophysical evidence for a significant positive association between sweet flavours and liking. This highly innovative application of two existing psychophysical measurement scales, which are typically used in the field of chemical senses, to tobacco flavour research can be expanded to examine the role of flavours in other flavoured tobacco products (eg, waterpipes, cigarillos, little cigars). Since tobacco products evoke various sensations and hedonic response just like any other foods and consumer products, the application of the scales in tobacco regulatory research holds great promise in better understanding the role of flavours in use behaviours. Additional studies are needed to further validate the scales and test their sensitivity in the tobacco research area.

Our findings of a significant positive association between sweet flavours and liking are consistent with previous studies, and thus not unexpected. A large proportion of adult e-cigarette users report adopting sweet flavours after e-cigarette initiation using tobacco-like flavours, ${ }^{10}$ and e-cigarette users in general report that they prefer sweet e-cigarette flavours. ${ }^{10} 14$ This study provides initial experimental evidence of the distinct and significant link between sweet flavours and liking in e-cigarettes, adding to our knowledge on the role of flavours in e-cigarette use and offering an enhanced understanding of the importance of sweet flavours.

\section{Limitations}

The present study has several limitations. Because of the small sample size, our study is underpowered to examine the roles of demographic characteristics and other tobacco/e-cigarette use characteristics on the relationships between sensory intensity and liking/disliking. One of the study eligibility criteria may have yielded a rather limited pool of study participants, notably the use of at least a 'medium' level $(\geq 9 \mathrm{mg} / \mathrm{mL})$ of nicotine strength was required for inclusion. Many respondents to the study cited the use of a 3-6 mg/mL nicotine e-liquid. Since our study was conducted with experienced e-cigarette users, our findings are not generalisable to first-time e-cigarette users, including both smokers and non-smokers, whose sensory attribute ratings and hedonic ratings would likely be different. In addition, our study was limited to adults with a large range in age. Further, our choice of e-cigarette, blu Tanks, might not be representative of popular e-cigarettes more often purchased. Therefore, the results of the study may not be generalisable across populations and e-cigarette devices. While all of the six regular flavours sold for blu Tanks were adopted for the study, these flavours do not represent the full variety and breadth of flavours available in the market.

\section{CONCLUSION}

This study provides psychophysical evidence of the significant role that flavours play in e-cigarette preference, and most likely use. More specifically, our findings suggest that sweet flavours enhance the appeal and liking of e-cigarette use. The greater attractiveness of e-cigarettes in terms of flavour presents the potential to reduce smoking in existing cigarette smokers but may also contribute to indirect harm by facilitating initiation of tobacco products and increasing the intensity of tobacco product use. If flavour is the major reason to initiate and continue to use e-cigarettes, especially for youth and young adults, ${ }^{36}$ more research is needed to determine which chemical compounds, and the specific concentration thresholds of those compounds, can influence the liking/disliking of e-cigarettes for specific populations of users. Systematic testing of these chemicals to determine the toxicity of the thermal degradation products generated and inhaled as a result of commercial ecigarette device-like conditions is called for. Finally, a consideration of the potential benefits of attractively flavoured ecigarettes as an aid for combustible tobacco users to either quit or reduce their smoking must be balanced against the concern that these flavoured e-cigarettes may lead to greater use initiation of a younger population.

\section{What this paper adds}

- This study applied psychophysical methods to measure sensory attributes of and hedonic responses to electronic cigarette (e-cigarette) flavours that are currently available in the market.

- This paper provides empirical evidence that perceived sweetness and coolness are positively correlated with liking of e-cigarette flavours that contain nicotine, whereas perceived bitterness and harshness are negatively correlated.

- Overall, bitterness and harshness, most likely from nicotine, reduce the liking of e-cigarette flavours, but sweetness and coolness significantly improve liking.

- The impact of sweetness on liking is greater than the impact of coolness in our study, implying that the role of sweet flavours in e-cigarettes is potentially a more significant factor in attracting sole e-cigarette users and dual e-cigarette and cigarette users.

Acknowledgements The authors thank Azieb Kidanu, MPH for her administrative support.

Contributors HK conceived and led the study, including the design, the data analyses and the writing up of the findings. JL advised on the study, analysed data, and provided key input into the interpretation and write up of study findings. SSB contributed to the study design, facilitated recruitment and laboratory processing of participants, and contributed to the interpretation and write up of study findings. MCB contributed to the study design, and contributed to the interpretation and write up of study findings. NMJ, LW and KSC processed laboratory participants and collected data. PIC advised on the study.

Funding Research reported in this publication was supported by grant number P50CA180523-S1 from the National Cancer Institute and FDA Center for Tobacco Products (CTP) awarded to the University of Maryland.

Disclaimer The content is solely the responsibility of the authors and does not necessarily represent the official views of the NIH or the Food and Drug Administration.

Competing interests None declared.

Patient consent Obtained.

Ethics approval Battelle Institutional Review Board.

Provenance and peer review Not commissioned; externally peer reviewed.

\section{REFERENCES}

1 Jaffe AJ, Glaros AG. Taste dimensions in cigarette discrimination: a multidimensional scaling approach. Addict Behav 1986;11:407-13.

2 King BA, Tynan MA, Dube SR, et al. Flavored-little-cigar and flavored-cigarette use among U.S. middle and high school students. J Adolesc Health 2014;54:40-6.

3 Carpenter CM, Wayne GF, Pauly JL, et al. New cigarette brands with flavors that appeal to youth: tobacco marketing strategies. Health Aff (Millwood) 2005;24:1601-10.

4 Manning KC, Kelly KJ, Comello ML. Flavoured cigarettes, sensation seeking and adolescents' perceptions of cigarette brands. Tob Control 2009;18:459-65.

5 U.S. Food and Drug Administration. (1 January 2011) Flavored Tobacco Product Sheet. http://www.fda.gov/downloads/TobaccoProducts/Labeling/ ProductsIngredientsComponents/UCM183215.pdf (accessed 15 Apr 15).

6 Dachille K. Pick your poison: responses to the marketing and sale of flavoured tobacco products. A Law Synopsis by the Tobacco Control Legal Consortium, 
February 2009. http://publichealthlawcenter.org/sites/default/files/resources/ tclc-syn-flavored-2009.pdf (accessed 15 Apr 2016).

7 H.R. 1256. Family Smoking Prevention \& Tobacco Control Act 2009. http://www. gpo.gov/fdsys/pkg/BILLS-111 hr1256enr/pdf/BILLS-111hr1256enr.pdf (accessed 15 Apr 2015).

8 US Food and Drug Administration, HHS. Deeming tobacco products to be subject to the Federal Food, Drug, and Cosmetic Act, as amended by the Family Smoking Prevention and Tobacco Control Act; restrictions on the sale and distribution of tobacco products and required warning statements for tobacco products. Final rule. Fed Regist 2016;81:28973-9106.

9 Zhu SH, Sun JY, Bonnevie E, et al. Four hundred and sixty brands of e-cigarettes and counting: implications for product regulation. Tob Control 2014;23(Suppl 3): iii3-9.

10 Farsalinos KE, Romagna G, Tsiapras D, et al. Impact of flavour variability on electronic cigarette use experience: an internet survey. Int I Environ Res Public Health 2013;10:7272-82.

11 Ambrose BK, Day HR, Rostron B, et al. Flavored tobacco product use among US youth aged 12-17 years, 2013-2014. JAMA 2015;314:1871-3.

12 Corey CG, Ambrose BK, Apelberg BJ, et al. Flavored tobacco product use among middle and high school students—United States, 2014. MMWR Morb Mortal Wkly Rep 2015;64:1066-70.

13 Feirman SP, Lock D, Cohen JE, et al. Flavored tobacco products in the United States: a systematic review assessing use and attitudes. Nicotine Tob Res 2016;18:739-49.

14 Krishnan-Sarin S, Morean ME, Camenga DR, et al. E-cigarette use among high school and middle school adolescents in Connecticut. Nicotine Tob Res 2015;17:810-18.

15 Berg CJ. Preferred flavors and reasons for e-cigarette use and discontinued use among never, current, and former smokers. Int J Public Health 2016;61:225-36.

16 Rosbrook K, Green BG. Sensory effects of menthol and nicotine in an e-cigarette. Nicotine Tob Res 2016;18:1588-95.

17 Crenshaw MD, Tefft ME, Buehler SS, et al. Determination of nicotine, glycerol, propylene glycol and water in electronic cigarette fluids using quantitative (1) H NMR. Magn Reson Chem. 2016. Published Online First: 21 Aug 2016. doi:10. $1002 /$ mrc. 4498

18 Lim J, Wood A, Green BG. Derivation and evaluation of a labeled hedonic scale. Chem Senses 2009;34:739-51.

19 Lim J, Fujimaru T. Evaluation of the labeled hedonic scale under different experimental conditions. Food Qual Pref 2010;21:521-30.

20 Lim J. Hedonic scaling: a review of methods and theory. Food Qual Pref 2011;22:733-47.
21 Green BG, Shaffer GS, Gilmore MM. Derivation and evaluation of a semantic scale of oral sensation magnitude with apparent ratio properties. Chem Senses 1993;18:683-702.

22 Green BG, Dalton P, Cowart B, et al. Evaluating the 'Labeled Magnitude Scale' for measuring sensations of taste and smell. Chem Senses 1996;21:323-34.

23 Bartoshuk LM, Duffy VB, Fast K, et al. Labeled scales (e.g., category, Likert, VAS) and invalid across-group comparisons: what we have learned from genetic variation in taste. Food Qual Pref 2002;14:125-38.

24 Foulds J, Veldheer S, Yingst J, et al. Development of a questionnaire for assessing dependence on electronic cigarettes among a large sample of ex-smoking E-cigarette users. Nicotine Tob Res 2015;17:186-92.

25 Beauchamp GK. Why do we like sweet taste: a bitter tale? Physiol Behav 2016;164 (Pt B):432-7.

26 Cowart BJ, Beauchamp GK, Mennella JA. Development of taste and smell in the neonate. In: Polin RA, Fox WW, Abman SH, eds. Fetal and neonatal physiology. Philadelphia: Elsevier Saunders, 2011:1899-907.

27 Carpenter CM, Wayne GF, Connolly GN. The role of sensory perception in the development and targeting of tobacco products. Addiction 2007;102:136-47.

28 Kreslake JM, Wayne GF, Alpert HR, et al. Tobacco industry control of menthol in cigarettes and targeting of adolescents and young adults. Am J Public Health 2008;98:1685-92.

29 Ogden MW, Characterization of menthol history and design of menthol cigarettes, presentation for Tobacco Products Scientific Advisory Committee, 15 July 2010 http://www.fda.gov/downloads/AdvisoryCommittees/CommitteesMeetingMaterials/ TobaccoProductsScientificAdvisoryCommittee/UCM220038.pdf (accessed 1 Apr 2016).

30 Klausner K. Menthol cigarettes and smoking initiation: a tobacco industry perspective. Tob Control 2011;20(Suppl 2):ii12-19.

31 Yerger VB, McCandless PM. Menthol sensory qualities and smoking topography: a review of tobacco industry documents. Tob Control 2011;20(Suppl 2):ii37-43.

32 Lawrence D, Cadman B, Hoffman AC. Sensory properties of menthol and smoking topography. Tob Induc Dis 2011;9(Suppl 1):S3.

33 Lee YO, Glantz SA. Menthol: putting the pieces together. Tob Control 2011;20 (Suppl 2):ii1-7.

34 Hersey JC, Ng SW, Nonnemaker JM, et al. Are menthol cigarettes a starter product for youth? Nicotine Tob Res 2006;8:403-13.

35 Villanti AC, Giovino GA, Barker DC, et al. Menthol brand switching among adolescents and young adults in the National Youth Smoking Cessation Survey. Am J Public Health 2012;102:1310-12.

36 Kong G, Morean ME, Cavallo DA, et al. Reasons for electronic cigarette experimentation and discontinuation among adolescents and young adults. Nicotine Tob Res 2015;17:847-54. 